\title{
Adipose-derived regenerative cells beneficial effects on systemic responses following myocardial ischemia/reperfusion
}

\author{
Masayuki Takamura, Soichiro Usui, Oto Inoue, Hiroshi Ootsuji, \\ Shin-ichiro Takashima, Ayano Nomura, Takeshi Kato, Hisayoshi Murai, \\ Hiroshi Furusho, Yoshio Sakai, Shuichi Kaneko \\ Department of Disease Control and Homeostasis, Kanazawa University Graduate \\ School of Medical Science, Kanazawa, Ishikawa, Japan
}

\begin{abstract}
Background: Acute coronary syndrome leads to systemic responses, including activation of the sympathetic nervous system, inflammation of atherosclerotic lesions, changes in metabolism and gene expressions of remote organs such as the spleen, bone marrow, and liver. Clinical trials and experimental studies have demonstrated that therapy with adipose-derived regenerative cells (ADRCs) attenuates myocardial ischemia/reperfusion $(I / R)$ injury. The aim of this study is to investigate the role of ADRCs in regulating systemic reactions following $I / R$. Methods: Isolated ADRCs were obtained from green fluorescent protein transgenic male mice. Flow cytometry revealed that freshly isolated ADRCs expressed stem cell markers CD90 and Sca-1, and mesenchymal lineage marker. These cells exhibited multilineage differentiation into adipogenic, osteogenic, and chondrogenic lineages. Wild-type mice were subjected to $30 \mathrm{~min}$ of left ascending coronary ischemia and $24 \mathrm{~h}$ reperfusion. Freshly isolated ADRCs $\left(10^{5}\right.$ cells) or vehicle (VEH), were administered intravenously through the tail at the time of reperfusion. Results: Compared to VEH, administration of ADRCs significantly reduced circulating troponin levels $24 \mathrm{~h}$ after I/R. Using quantitative real-time polymerase chain reaction analysis, the present study confirms that I/R-induced increase of factor $X m R N A$ expression in the liver and was significantly inhibited by ADRCs compared to VEH. Administration of ADRCs significantly reduced the $I / R$-induced increase in serum levels of the proinflammatory cytokines tumor necrosis factor-alpha and interleukin-18 seen in mice receiving VEH.

Conclusions: These results suggest that administration of ADRCs could have an important role in reducing myocardial injury and regulating the hepatic gene expression profile following $I / R$. (Cardiol J 2016; 23, 6: 685-693)
\end{abstract}

Key words: adipose-derived regenerative cells, myocardial ischemia/reperfusion, cytokine, liver, factor $\mathrm{X}$

Address for correspondence: Soichiro Usui, MD, PhD, Department of Disease Control and Homeostasis,

Kanazawa University Graduate School of Medical Science, 13-1 Takara-machi, Kanazawa, Ishikawa, 920-8641, Japan, tel: +81-76-265-2238, fax: +81-76-234-4250, e-mail: usuiso@m-kanazawa.jp 


\section{Introduction}

Ischemic heart diseases including acute myocardial infarction (MI), are some of the leading causes of morbidity and mortality worldwide [1]. Regenerative cell therapies with bone-marrowderived mesenchymal stromal cells (MSCs) have demonstrated an ability to improve cardiac function and remodeling in clinical trials [2]. These studies, however, did not demonstrate that MSCs were able to differentiate into cardiac myocytes, or to engraft and replace necrotic myocardium. It has been postulated that MSCs act via indirect mechanisms other than cell engraftment, such as inhibition of inflammation, cytokine production, and angiogenesis in promoting cardiac repair $[3,4]$.

Recent studies have demonstrated that the stromal vascular fraction of the adipose tissue contains mesenchymal stem cells termed adiposederived regenerative cells (ADRCs), not only have a capacity for multipotent differentiation, but are also able to produce a wide array of growth factors and cytokines $[5,6]$. Compared to bone marrow, subcutaneous adipose tissue represents an effective source of regenerative therapy because of its abundance, surgical accessibility and high content of multipotent mesenchymal progenitor cells [7]. Acute MI is accompanied by systemic changes in inflammation, coagulation, and metabolism, which may affect the outcome and prognosis of an acute coronary syndrome. Indeed, there are some systemic reactions involving multiple organs, which may exacerbate the cardiac events. A previous study has shown that hepatic gene expression, coagulation factors, and protein $\mathrm{C}$, have levels which fluctuate during acute $\mathrm{MI}$ and are potentially regulated by humoral factors of cardiac origin provoked by myocardial ischemia [8]. Although the administration of ADRCs improves cardiac function during myocardial ischemia/reperfusion ( $/ / \mathrm{R})$ injury [9], via secreted paracrine factors that attenuate inflammation and promote cell survival, the effect of ADRC administration on the systemic response to myocardial $\mathrm{I} / \mathrm{R}$ requires further study.

The aim of the present study is to clarify whether the administration of ADRCs affects the systemic response following myocardial I/R.

\section{Methods}

\section{Ethics statement}

All animal protocols were approved by and performed in accordance with Guide for the Care and Use of Laboratory Animals issued by Kanazawa
University, which strictly conforms to Guide for the Care and Use of Laboratory Animals, published by the US National Institutes of Health (NIH, Bethesda, MD).

\section{Isolation of adipose-derived regenerative cells}

The cells making up the adipose stroma were isolated adhering to a previously described protocol with slight modifications [10]. Briefly, adipose tissue was obtained from the inguinal subcutaneous region of 10 -week-old non-transgenic (nTg) green fluorescent protein transgenic (GFP-Tg) male mice (a gift from Professor Okabe, Osaka University, Japan). After all lymph nodes had been removed, the adipose tissue was washed three times with equal volumes of phosphate buffered saline (PBS)(-), and incubated with Liberase TM Research Grade (Roche, Mannheim, Germany). The digested cells were centrifuged resuspended in PBS(-) and were then filtered with a mesh.

\section{Cell culture and multilineage differentiating potential}

Cells were cultured under standard cell culture conditions with Dullbecco's modified Eagele's medium (DMEM)/Nutrient Mixture F-12 1:1 medium (Life Technologies-Invitrogen, Carlsbad, CA, USA) supplemented with $10 \%$ heat-inactivated fetal bovine serum and $1 \%$ antibiotic-antimycotic solution (Life Technologies). The pluripotency of ADRCs was examined using a mouse mesenchymal stem cell functional kit (R\&D systems, Minneapolis, MN, USA) [11]. Differentiation into adipocytes was detected by oil red $\mathrm{O}$ staining after 14 days of incubation in the presence of adipocyte induction factors (hydrocortisone, isobuthlmethylxanthine, and indomethacin). Differentiation into osteocytes was detected by alkaline phosphatase staining (Nichirei, Tokyo, Japan), after 14 days of incubation in Osteogenic Differentiation Medium. To induce chondrogenic differentiation, the cell pellets were cultured in $15 \mathrm{~mL}$ conical tubes. After 21 days of incubation with Chondrogenic Differntiation Medium, pellets were harvested, washed in $4 \%$ paraformaldehyde, and stained with toluidine blue (Wako, Tokyo, Japan).

\section{Cell-surface markers}

Cell-surface markers on fleshly isolatedADRCs were evaluated by flow cytometry using the following antibodies [12]: fluorescein isothiocyanate (FITC)-conjugated anti-CD45 antibody, a marker of bone-marrow-derived cells and anti- 
CD31 antibody, a marker of vascular endothelial cells; phycoerythrin (PE)-conjugated CD34 antibody, a marker of endothelial progenitor cells; Sca-1, CD44 and allophycocyanin (APC)-conjugated CD90 antibodies, markers of mesenchymal stem cells; and the corresponding isotype controls. All antibodies were purchased from BD Pharmingen (BD Biosciences, San Jose, CA, USA). Flow cytometry analysis was performed using a FACS Accuri C6 (BD Biosciences) cytometer and analyzed with FlowJo (Tree Star, Ashland, OR, USA).

\section{Myocardial ischemia/reperfusion}

Male C57BL/6J mice (Charles River Laboratories, Yokohama, Japan) were subjected to I/R in a sham operation, as described elsewhere [13]. A lateral thoracotomy was performed in the anesthetized and ventilated mice, and a 7-0 suture was looped under the left descending coronary artery and used to induce coronary artery occlusion for $30 \mathrm{~min}$, followed by $24 \mathrm{~h}$ of reperfusion. A total of $10^{5}$ cells in a volume of $0.2 \mathrm{~mL}$ PBS per animal was administered intravenously through the tail vein after reperfusion. The hearts were removed, and the area at risk (AAR) and infarct area (IA) were determined using staining with Evans blue and 2,3,5-triphenyltetrazolium chloride.

\section{Flow cytometry}

The distribution of GFP-positive cells in each organ was analyzed by flow cytometry [14]. Each organ tissue was cut into pieces and treated with Liberase at $37^{\circ} \mathrm{C}$ for $30-60 \mathrm{~min}$ with gentle agitation. The cells were washed with MACS ${ }^{\mathrm{TM}}$ buffer (PBS, $0.5 \%$ BSA, 2 mM EDTA, degassed) twice and filtered with a $40-\mu \mathrm{m}$ cell strainer for single cell suspension.

\section{Serum troponin I measurement}

Blood samples were collected $24 \mathrm{~h}$ after I/R. Cardiac troponin I (TnI) levels were measured in serum using a mouse cardiac TnI ELISA kit (Life Diagnostics, Inc, West Chester, PA, USA) in accordance with manufacturer protocols.

\section{Isolation of mRNA and quantitative real-time polymerase chain reaction \\ Total RNA was isolated using an RNA isolation kit (Sigma-Aldrich) in accordance with manufactu- rer protocols. Total RNA (100 ng) was used to gene- rate cDNA using the TaqMan Universal Master Mix (Applied Biosystems, Foster City, CA, USA), in accordance with manufacturer protocols [8]. Quantitative real-time polymerase chain reaction (qRT-PCR) analysis was performed using the ABI}

Prism 7300 sequence detection system (Applied Biosystems). The following primers and TaqMan probes (Applied Biosystems) were used: protein C (ProC Mm00435966_m1), factor X (F10 Mm00484177_m1), CNTF(CNTFMm00446373_m1), and CSF1 (CSF1, Mm 00432686 m1). Beta actin (Actb, Mm02619580_g1) was use-d as an endogenous control.

\section{Measurement of cytokines and chemokines \\ Tumor necrosis factor (TNF)- $\alpha$, interleukin} (IL)-6, IL-1 $\beta$, IL-18, macrophage inflammatory protein (MIP)-1 $\alpha$, granulocyte-colony-stimulating factor (GCSF), regulated on activation normal $T$ cells expressed and secreted (RANTES), granulocyte macrophage colony-stimulating factor (GMCSF), and monocyte chemoattractant protein (MCP)- 1 levels in serum were monitored using the Bio-Plex Cytokine Assay System (Bio-Rad Laboratories, Hercules, CA, USA), in accordance with manufacturer protocols [12].

\section{Western blot analysis}

Tissue homogenates were prepared using RIPA buffer (Upstate). Antibody against factor X obtained from Thermo Fisher Scientific. Antibody against glyceraldehyde-3-phosphate dehydrogenase (GAPDH) obtained from Santa-Cruz Biotechnologies.

\section{Statistics}

All values are expressed as mean \pm standard error of mean. Statistical analyses performed using either t-test or one-way ANOVA followed by a post hoc Bonferroni-Dunn comparison test. A value of $p<0.05$ was considered significant. Statistical analyses were conducted using GraphPad Prism (GraphPad Software, La Jolla, CA).

\section{Results}

\section{Characterization of ADRCs}

To understand the characteristics of the ADRCs, cell surface markers were analyzed. The percentages of Sca- 1 and CD90, markers of stem cells, were $47.0 \%$ and $39.2 \%$, respectively, of the total nucleated cells (Fig. 1A, B). One-fourth of total nucleated cells expressed CD44, the mesenchymal linage marker (Fig. 1C) CD45, a marker of hematopoietic cells and leukocytes CD31, an endothelial cell marker CD34, a myeloid progenitor antigen made up $25.1 \%, 5.2 \%$ and $0.8 \%$ respectively of the total nucleated cells (Fig. 1D, E, F).

To determine the multilineage differentiation of ADRCs their differentiation into adipogenic, 


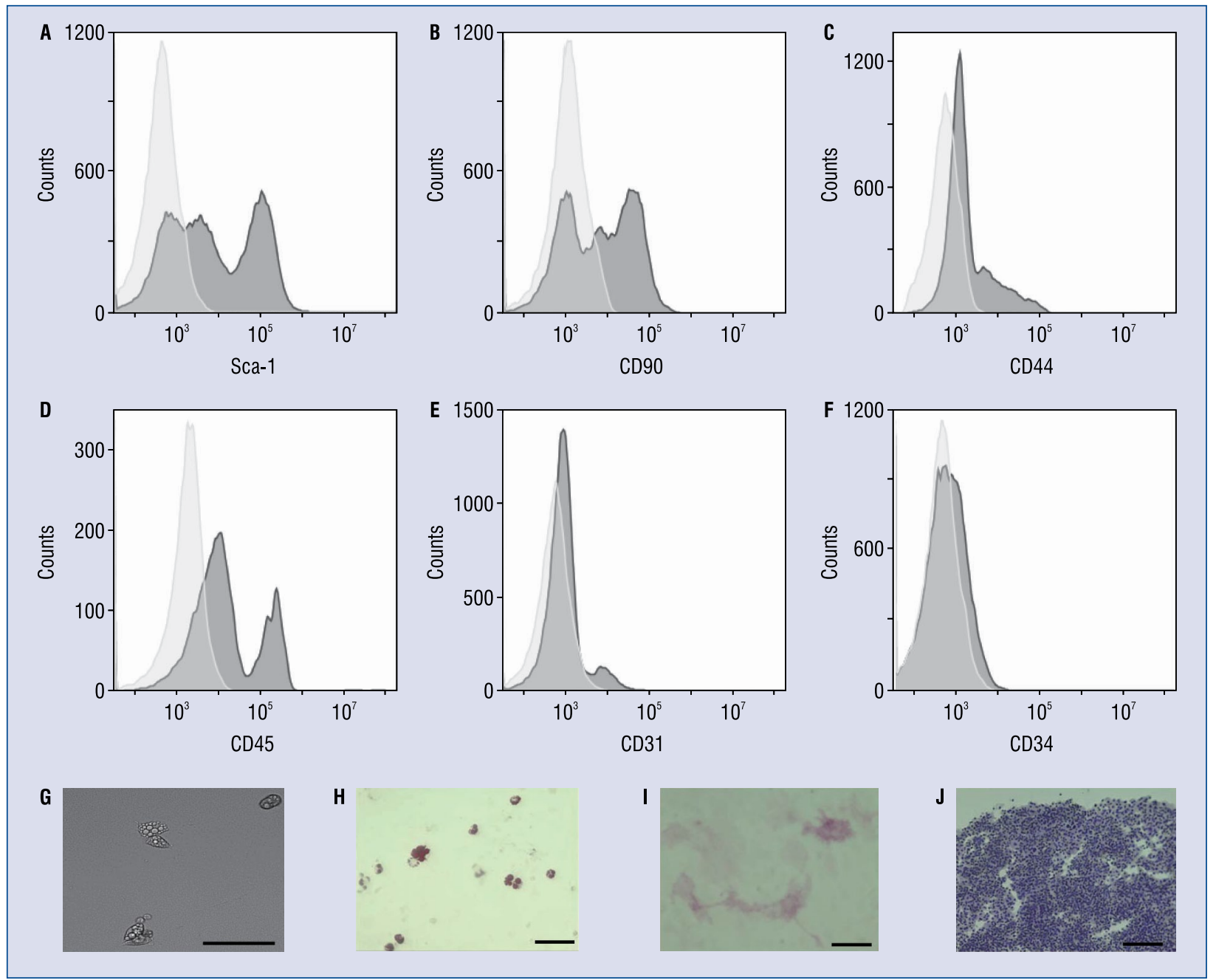

Figure 1. Characteristics and pluripotency of adipose-derived regenerative cells (ADRCs). ADRCs had stem-cellsurface markers. Fluorescence-activated cell sorting analysis of ADRCs. The shaded areas represent cells labeled with Sca-1 (A), CD90 (B), CD44 (C), CD45 (D), CD31 (E), and CD34 (F). The white areas demonstrate negative controls (isotype controls). ADSCs had multipotency of differentiation. The cells had lipid droplets (G) and were positive for oil red $\mathrm{O}$ stain $\mathbf{( H )}$ under the specific stimulation for fat. The cells were positive for alkaline phosphatase under the specific stimulation for osteocyte (I). The cells were positive for Alcian Blue stain under the specific stimulation for chondrocyte (J). Scale bar, $100 \mu \mathrm{m}$ for each panel.

osteogenic, and chondrogenic lineages was examined. After 7 days of incubation in the presence of adipogenic differentiation induction factor, fat droplets were detected in cell cytoplasm (Fig. 1G). These cells were stained with oil red O (Fig. 1H). The differentiation of osteocytes was detected by alizarin red stain for matrix mineralization (Fig. 1I) 14 days after incubation in the presence of differentiation factors for osteocytes. To determine differentiation into chondrocytes, ADRCs were incubated under chondrogenic conditions for 14 days.
The differentiation of chondrocytes was detected by toluidine blue stain (Fig. 1J).

\section{Distribution of administered ADRCs in $\mathbf{I} / \mathbf{R}$ models}

To investigate the influence of $\mathrm{I} / \mathrm{R}$ on the distribution of intravenously administered ADRCs in organs, I/R with sham operated mice were compared. Flow cytometry analysis demonstrated that GFP-positive ADRC infusion into I/R mice resulted in greater distribution in the heart and liver than 


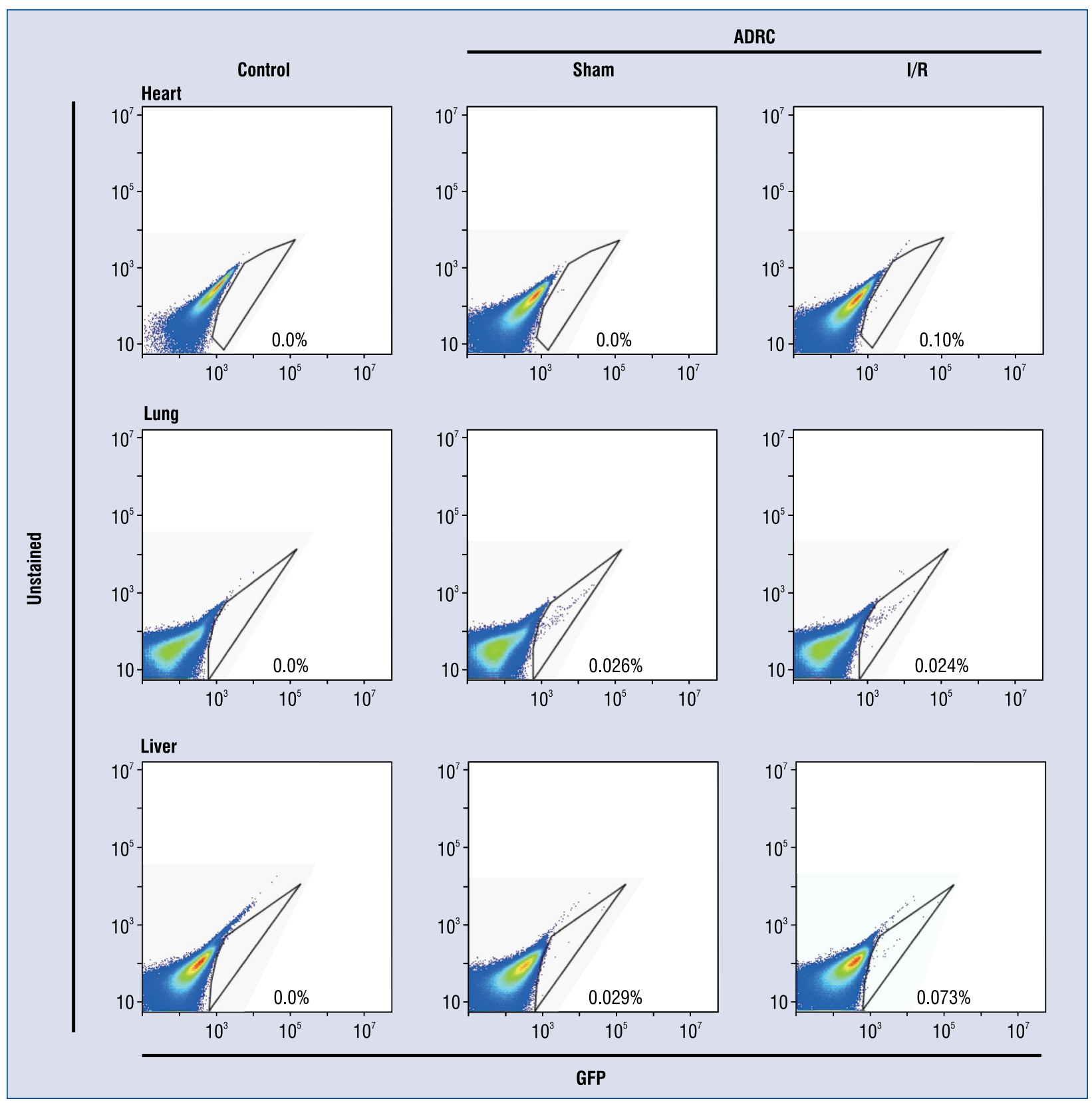

Figure 2. Distribution of adipose-derived regenerative cells (ADRCs) $24 \mathrm{~h}$ after intravenous injection. Flow cytometory analysis of green fluorescent protein (GFP)-positive cells in indicated organs after injection of ADRCs from GFP transgenic mice; l/R - myocardial ischemia/reperfusion.

in sham operated mice (Fig. 2). There was no difference in the distribution of ADRCs in lung tissue between $\mathrm{I} / \mathrm{R}$ mice and sham operated mice.

\section{$\mathrm{I} / \mathrm{R}$ injury is ameliorated} by administered ADRCs

To examine the effect of administration of ADRCs during I/R injury, 30 min of ischemia and subsequent $24 \mathrm{~h}$ of reperfusion to $\mathrm{I} / \mathrm{R}$ mice was applied. The mean AAR in the left ventricular area was not similar in the ADRCs group and the vehicle (VEH) (Fig. 3A, B). The administration of ADRCs significantly attenuated I/R-induced increases in IA/AAR (Fig. 3C). Furthermore, it was observed that administration of ADRCs significantly reduced circulating troponin levels in the ADRCs group 


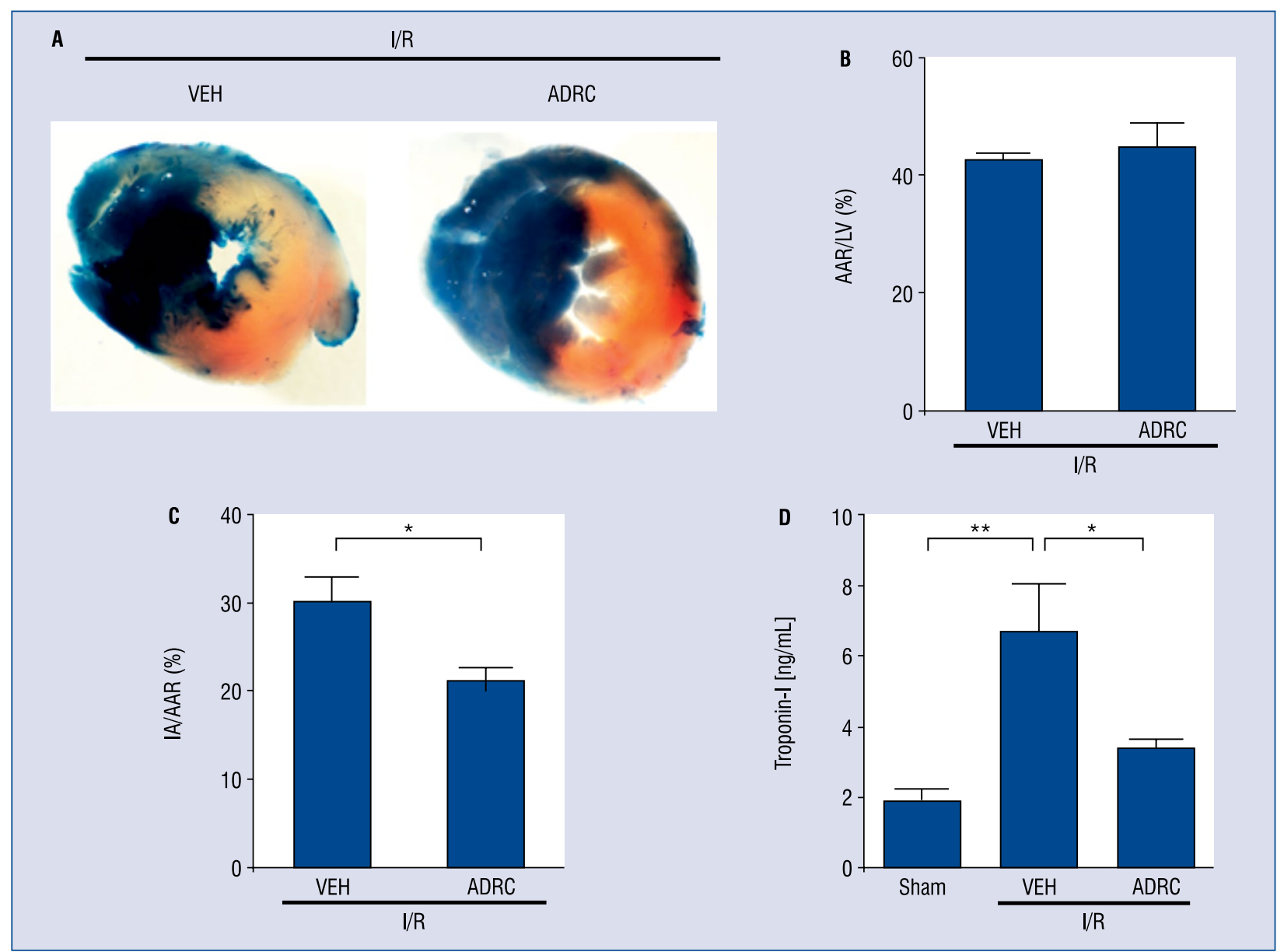

Figure 3. Administration of adipose-derived regenerative cells (ADRCs) inhibits myocardial ischemia/reperfusion $(\mathrm{I} / \mathrm{R})$ induced myocardial damage. Representative examples of myocardial infarction stained with Evans Blue (EB) and triphenyl tetrazolium chloride (TTC) $24 \mathrm{~h}$ after reperfusion (A). The area at risk (AAR) was expressed as a percentage of the left ventricle (LV) (B). Myocardial infarct area (IA) expressed as a percentage of AAR (C). Troponin I levels in vehicle (VEH)- and ADRCs-treated mice at $24 \mathrm{~h}$ after reperfusion (D).

to $3.34 \pm 0.31 \mathrm{ng} / \mathrm{mL}$ compared with the $\mathrm{VEH}$ $(6.58 \pm 1.34 \mathrm{ng} / \mathrm{mL})$ (Fig. 3D).

\section{ADRC treatment alters I/R-induced} hepatic gene expression

It has been previously shown that myocardial $\mathrm{I} / \mathrm{R}$ affects gene expression profile in the liver, this is a remote organ having no changes in hemodynamic state [8]. Likewise, in this study qRT-PCR analysis demonstrated that the mRNA expression levels of ProC, factor X, and CNTF in the liver were significantly increased in the VEH group $24 \mathrm{~h}$ after I/R (Fig. 4A-C). I/R-induced upregulation of factor $\mathrm{X}$ expression in the liver was significantly inhibited in the ADRC-treated group (Fig. 4B). I/R-induced increases in ProC and
CNTF mRNA expression levels in the liver were not observed in the treatment group (Fig. 4A, C). Protein expression of factor $\mathrm{X}$ was also increased in the VEH and inhibited in the ADRC-treated group (Fig. 4E).

\section{ADRCs treatment reduces I/R-induced cytokine concentration in serum}

Cytokine levels in the serum were measured $24 \mathrm{~h}$ after I/R. Serum concentrations of TNF- $\alpha$ and IL-18 were significantly elevated in VEH group $24 \mathrm{~h}$ after I/R compared with those in sham-operated mice (Fig. 5A, D). Increases in TNF- $\alpha$ and IL-18 expression levels were significantly suppressed in the ADRCs-treated group when compared with those in the VEH group. 


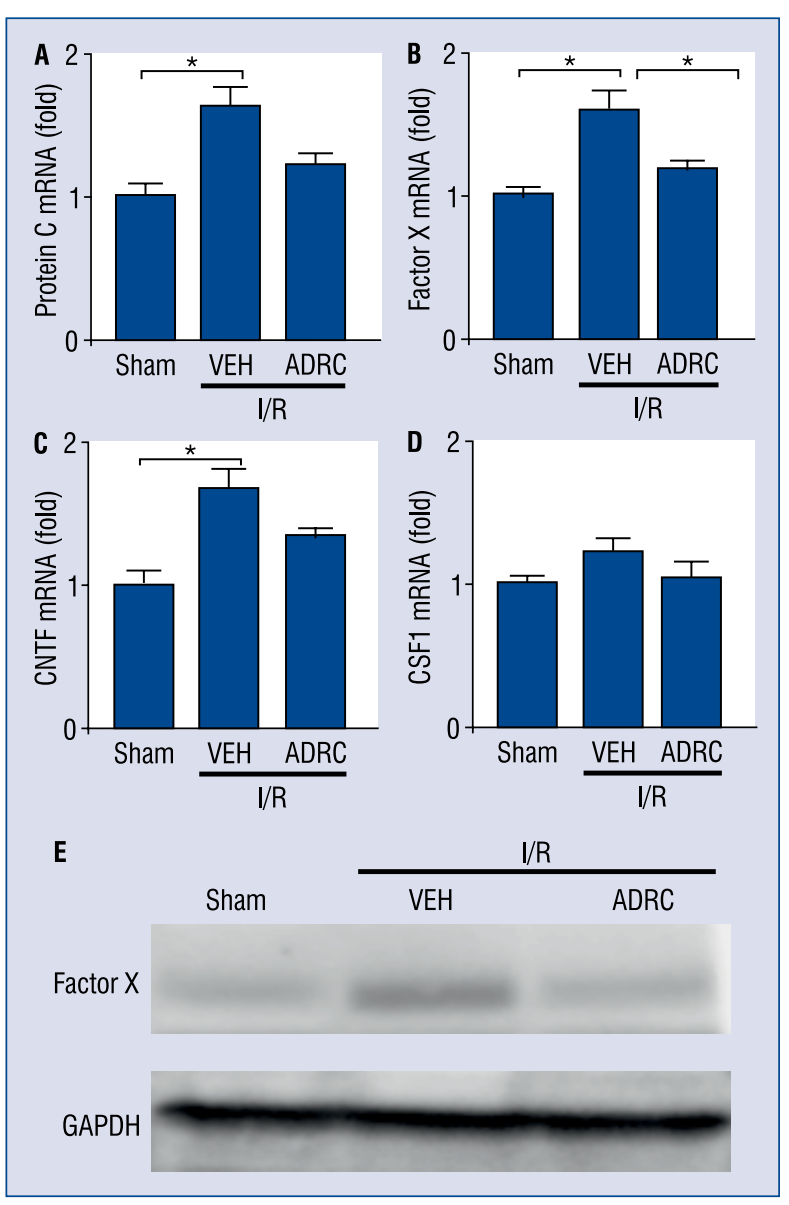

Figure 4. Administration of adipose-derived regenerative cells (ADRCs) affects gene expression in liver at $24 \mathrm{~h}$ after myocardial ischemia/reperfusion (I/R). Protein $C(\mathbf{A})$, coagulation factor $X$ (Factor $X)(B)$, ciliary neurotrophic factor (CNTF) (C), colony-stimulating factor-1 (CSF1) (D). Protein expression of factor $X$ and glyceraldehyde3-phosphate dehydrogenase (GAPDH) was determined by immunoblotting (F).

\section{Discussion}

The current study demonstrated an ameliorative effect of $\mathrm{ADRC}$ therapy on myocardial $\mathrm{I} / \mathrm{R}$ injury in the mouse heart. It has been shown that freshly isolated ADRCs, delivered acutely following ischemia and reperfusion, were able to protect against myocardial damage and regulate systemic responses following $\mathrm{I} / \mathrm{R}$. While no substantial number of ADRCs was detected in the infarcted heart, infused ADRCs were mainly detected in the lung and liver, intravenous systemic administration of $\mathrm{ADRCs}$ resulted in a reduction of proinflammatory cytokines in serum and affected hepatic gene expression profiles following I/R. These results suggest that not only engrafted ADRCs, but also
ADRCs trapped in the lung and liver, exert a protective effect following I/R injury.

Clinical trials have shown that cell therapy using ADRCs is safe and effective in reducing the damage to ischemic myocardium without significant engraftment [15]. The intravenous systemic administration of stem cells leads to entrapment in the lung and liver [16]. Although intravenous infusion results in poor engraftment in the heart, ADRCs are known to secrete numerous angiogenic, arteriogenic, chemotactic, and anti-apoptotic growth factors [3]. It appears that a paracrine signaling mechanism may be responsible for their favorable effects [17]. In this study, the distribution of freshly isolated ADRCs, delivered just shortly following left anterior descending artery occlusion and reperfusion, was low in the infarcted heart, but the administration of ADRCs did reduce myocardial injury. Similarly, Lee et al. [18] reported that the administered cells were distributed not in the heart but were detected temporarily in the lung, and had anti-inflammatory effects on the remote organ mediated by a humoral factor, such as TNF- $\alpha$ -stimulated gene-6. These findings suggest that the treatment of acute MI with freshly isolated ADRCs is a promising approach.

Several lines of evidence suggest that patients with nonalcoholic fatty liver disease have a high risk of developing cardiovascular diseases [19, 20], and it is reasonable to speculate that the liver is involved in a systemic reaction that modifies the pathogenesis of acute coronary syndrome. In this study, administration of ADRCs reduced myocardial $\mathrm{I} / \mathrm{R}$-induced upregulation of factor $\mathrm{Xa}$ in the liver. Inhibition of factor Xa has been shown to reduce inflammation in a model of acute myocardial I/R injury [21]. This protective effect has been associated with phosphorylation of ERK1/2 and STAT-3 [22, 23]. These results support the hypothesis that reduction of factor Xa expression in the liver may be one of the cardiprotective effects caused by ADRC administration. The acute-phase response presents disturbances of homeostasis due to infection, tissue injury, or immunologic disorders consisting of an initial local reaction at the site of injury and subsequent systemic reaction. An important part of the systemic reaction following cardiac injury is the stimulation of protein synthesis in the liver, such as protein $\mathrm{C}$ and $\mathrm{CNTF}$ [8]. In this study, I/R-induced upregulation of CNTF and protein $\mathrm{C}$ in the liver was not observed. This result is dependant upon the reduction of myocardial I/R-induced liver damage by the administration of ADRCs. Further 


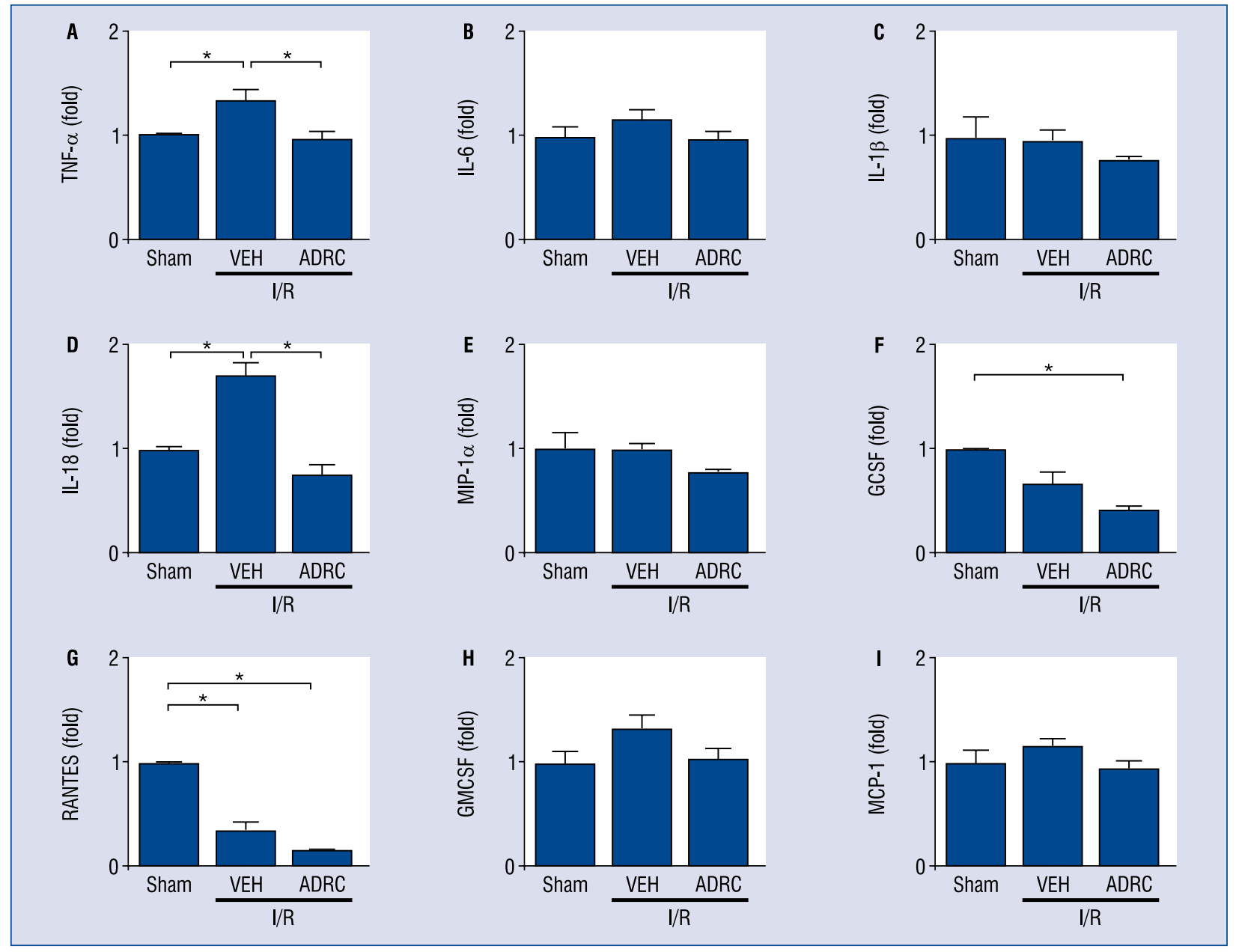

Figure 5. Administration of adipose-derived regenerative cells (ADRCs) affects some inflammatory cytokine levels in serum at $24 \mathrm{~h}$ after myocardial ischemia/reperfusion (I/R). Serum samples were detected by the Bio-Plex Cytokine Assay System. Tumor necrosis factor (TNF)- $\alpha$ (A), interleukin (IL)-6 (B), IL-1 $\beta$ (C), IL-18 (D), macrophage inflammatory protein (MIP)-1 $\alpha$ (E), granulocyte-colony-stimulating factor (GCSF) (F), regulated upon activation of normal T cells that express and secrete (RANTES) (G), granulocyte macrophage colony-stimulating factor (GMCSF) (H), monocyte chemoattractant protein (MCP)-1 (I); VEH - vehicle.

studies are needed to reveal the role of these protein expressions in the liver during myocardial I/R.

A growing body of evidence indicates a relatively low rate of stem cell differentiation into cardiomyocytes following transplantation, suggesting that the anti-inflammatory effect is one of the major mechanisms that mediate cardioprotection $[24,25]$. Furthermore, in experimental models, proinflammatory cytokines, such as TNF- $\alpha$ and IL-18 [26], have been shown to significantly increase cardiac rupture and dysfunction through an increase in myocyte apoptosis and infarct size. In this study, it has been demonstrated that the expression of TNF- $\alpha$ and IL-18 was induced following cardiac I/R. The increase in blood TNF- $\alpha$ levels, as a consequence of overproduction of cytokine in the border zone of the infarct, which is strongly correlated with deleterious effects including left ventricular dysfunction [27]. High serum levels of IL-18 were also associated with an increased risk of developing cardiovascular disease in acute MI patients [28]. The pathogenic actions of TNF- $\alpha$, and IL-18 have been implicated in myocardial I/R injury and remodeling. Thus, the reduction in the levels of these proinflammatory mediators induced by $\mathrm{ADRC}$ administration may explain the favorable effects on I/R injury.

\section{Conclusions}

The results of this study show that the administration of freshly isolated ADRCs could have 
a protective effect in reducing myocardial damage following I/R, even without engraftment. New insight is offered into these protective mechanisms and suggest that they involve changes in systemic responses, including hepatic gene expression profiles.

\section{Acknowledgements}

This research was supported by Grants-in-Aid for Scientific Research (C-25461049 to MT, C-15K09135 to SU), and a Grant-in-aid for Young Scientists (B-26860549 to ST) from the Ministry of Education, Culture, Sports, Science, and Technology of Japan.

\section{Conflict of interest: None declared}

\section{References}

1. Weaver WD, Simes RJ, Betriu A et al. Comparison of primary coronary angioplasty and intravenous thrombolytic therapy for acute myocardial infarction: a quantitative review. JAMA, 1997; 278: 2093-2098.

2. Takashima S, Tempel D, Duckers HJ. Current outlook of cardiac stem cell therapy towards a clinical application. Heart, 2013; 99: 1772-1784.

3. Gnecchi M, Zhang Z, Ni A, Dzau VJ. Paracrine mechanisms in adult stem cell signaling and therapy. Circ Res, 2008; 103: 1204-1219.

4. Chen JJ and Zhou SH. Mesenchymal stem cells overexpressing MiR-126 enhance ischemic angiogenesis via the AKT/ERK-related pathway. Cardiol J, 2011; 18: 675-681.

5. Meliga E, Strem BM, Duckers HJ, Serruys PW. Adipose-derived cells. Cell Transplant, 2007; 16: 963-970.

6. Zuk PA, Zhu M, Ashjian P et al. Human adipose tissue is a source of multipotent stem cells. Mol Biol Cell, 2002; 13: 4279-4295.

7. Rasmussen JG, Frobert O, Holst-Hansen $\mathrm{C}$ et al. Comparison of human adipose-derived stem cells and bone marrow-derived stem cells in a myocardial infarction model. Cell Transplant, 2014; 23: 195-206.

8. Ootsuji H, Honda M, Kaneko S et al. Altered hepatic gene expression profiles associated with myocardial ischemia. Circ Cardiovasc Genet, 2010; 3: 68-77.

9. Bagno LL, Werneck-de-Castro JP, Oliveira PF et al. Adiposederived stromal cell therapy improves cardiac function after coronary occlusion in rats. Cell Transplant, 2012; 21: 1985-1996.

10. Seki A, Sakai Y, Komura T et al. Adipose tissue-derived stem cells as a regenerative therapy for a mouse steatohepatitisinduced cirrhosis model. Hepatology, 2013; 58: 1133-1142.

11. Higashimoto M, Sakai Y, Takamura M et al. Adipose tissue derived stromal stem cell therapy in murine ConA-derived hepatitis is dependent on myeloid-lineage and CD4+ T-cell suppression. Eur J Immunol, 2013; 43: 2956-2968.

12. Furuichi K, Shintani H, Sakai $Y$ et al. Effects of adipose-derived mesenchymal cells on ischemia-reperfusion injury in kidney. Clin Exp Nephrol, 2012; 16: 679-689.
13. Ohtani $\mathrm{K}$, Usui $\mathrm{S}$, Kaneko $\mathrm{S}$ et al. Benidipine reduces ischemia reperfusion-induced systemic oxidative stress through suppression of aldosterone production in mice. Hypertens Res, 2012; 35: 287-294.

14. Kitano K, Usui S, Ootsuji $\mathrm{H}$ et al. Rho-kinase activation in leukocytes plays a pivotal role in myocardial ischemia/reperfusion injury. PLoS One, 2014; 9: e92242.

15. Hare JM, Traverse JH, Henry TD et al. A randomized, doubleblind, placebo-controlled, dose-escalation study of intravenous adult human mesenchymal stem cells (prochymal) after acute myocardial infarction. J Am Coll Cardiol, 2009; 54: 2277-2286.

16. Barbash IM, Chouraqui P, Baron J et al. Systemic delivery of bone marrow-derived mesenchymal stem cells to the infarcted myocardium: feasibility, cell migration, and body distribution. Circulation, 2003; 108: 863-868.

17. Oh JY, Kim MK, Shin MS et al. The anti-inflammatory and antiangiogenic role of mesenchymal stem cells in corneal wound healing following chemical injury. Stem Cells, 2008; 26: 1047-1055 .

18. Lee RH, Pulin AA, Seo MJ et al. Intravenous hMSCs improve myocardial infarction in mice because cells embolized in lung are activated to secrete the anti-inflammatory protein TSG-6. Cell Stem Cell, 2009; 5: 54-63.

19. Boddi $\mathrm{M}$, Tarquini $\mathrm{R}$, Chiostri $\mathrm{M}$ et al. Nonalcoholic fatty liver in nondiabetic patients with acute coronary syndromes. Eur J Clin Invest, 2013; 43: 429-438.

20. Guleria A, Duseja A, Kalra N, Das A, Dhiman R, Chawla Y, Bhansali A. Patients with non-alcoholic fatty liver disease (NAFLD) have an increased risk of atherosclerosis and cardiovascular disease. Trop Gastroenterol, 2013; 34: 74-82.

21. Macchi L, Moussa WB, Guillou S et al. The synthetic pentasaccharide fondaparinux attenuates myocardial ischemia-reperfusion injury in rats via STAT-3. Shock, 2014; 41: 166-171.

22. Pawlinski R, Tencati M, Hampton CR et al. Protease-activated receptor-1 contributes to cardiac remodeling and hypertrophy. Circulation, 2007; 116: 2298-2306.

23. Loubele ST, Spek CA, Leenders P et al. Activated protein C protects against myocardial ischemia/ reperfusion injury via inhibition of apoptosis and inflammation. Arterioscler Thromb Vasc Biol, 2009; 29: 1087-1092.

24. Bagno LL, Carvalho D, Mesquita F et al. Sustained IGF-1 secretion by adipose-derived stem cell improves infarcted heart function. Cell Transplant, 2016; 25: 1609-1622.

25. Rehman J, Traktuev D, Li J et al. Secretion of angiogenic and antiapoptotic factors by human adipose stromal cells. Circulation, 2004; 109: 1292-1298.

26. Venkatachalam K, Prabhu SD, Reddy VS, Boylston WH, Valente AJ, Chandrasekar B. Neutralization of interleukin-18 ameliorates ischemia/reperfusion-induced myocardial injury. J Biol Chem, 2009; 284: 7853-7865.

27. Sun M, Dawood F, Wen WH et al. Excessive tumor necrosis factor activation after infarction contributes to susceptibility of myocardial rupture and left ventricular dysfunction. Circulation, 2004; 110: 3221-3228.

28. Mallat $Z$, Henry P, Fressonnet $\mathrm{R}$ et al. Increased plasma concentrations of interleukin-18 in acute coronary syndromes. Heart, 2002; 88: 467-469. 